\title{
Beaches of islands of tractability: Algorithms for parsimony and minimum perfect phylogeny haplotyping problems ${ }^{\star}$
}

\author{
Leo van Iersel ${ }^{1}$, Judith Keijsper ${ }^{1}$, Steven Kelk ${ }^{2}$ and Leen Stougie ${ }^{12}$ \\ 1 Technische Universiteit Eindhoven (TU/e), Den Dolech 2, 5612 AX Eindhoven, \\ Netherlands, \\ l.j.j.v.iersel@tue.nl, j.c.m.keijsper@tue.nl, \\ http://www.tue.nl \\ 2 Centrum voor Wiskunde en Informatica (CWI), Kruislaan 413, 1098 SJ \\ Amsterdam, Netherlands, \\ steven.kelk@cwi.nl, leen.stougie@cwi.nl, \\ http://www. cwi.nl
}

\begin{abstract}
The problem Parsimony Haplotyping $(P H)$ asks for the smallest set of haplotypes which can explain a given set of genotypes, and the problem Minimum Perfect Phylogeny Haplotyping (MPPH) asks for the smallest such set which also allows the haplotypes to be embedded in a perfect phylogeny evolutionary tree, a well-known biologically-motivated data structure. For $P H$ we extend recent work of [16] by further mapping the interface between "easy" and "hard" instances, within the framework of $(k, l)$-bounded instances. By exploring, in the same way, the tractability frontier of $M P P H$ we provide the first concrete, positive results for this problem, and the algorithms underpinning these results offer new insights about how $M P P H$ might be further tackled in the future. In both $P H$ and $M P P H$ intriguing open problems remain.
\end{abstract}

\section{Introduction}

The computational problem of inferring biologically-meaningful haplotype data from the genotype data of a population continues to generate considerable interest at the interface of biology and computer science/mathematics. A popular underlying abstraction for this model (in the context of diploid organisms) represents a genotype as a string over a $\{0,1,2\}$ alphabet, and a haplotype as a string over $\{0,1\}$. The precise goal depends on the biological model being applied but a common, minimal algorithmic requirement is that, given a set of genotypes, a set of haplotypes must be produced which resolves the genotypes.

In this paper we focus on two different models. The first model, the parsimony haplotyping (PH) model [10], asks for a smallest (i.e. most parsimonious) set of haplotypes to resolve the input genotypes. To be precise, we are given a genotype

\footnotetext{
* Supported by the Dutch BSIK/BRICKS project
} 
matrix $G$ with elements in $\{0,1,2\}$, the rows of which correspond to genotypes, while its columns correspond to sites on the genome, called SNP's. A haplotype matrix has elements from $\{0,1\}$, and rows corresponding to haplotypes. Haplotype matrix $H$ resolves genotype matrix $G$ if for each row $g_{i}$ of $G$, containing at least one 2 , there are two rows $h_{i_{1}}$ and $h_{i_{2}}$ of $H$, such that $g_{i}(j)=h_{i_{1}}(j)$ for all $j$ with $h_{i_{1}}(j)=h_{i_{2}}(j)$ and $g_{i}(j)=2$ otherwise, in which case we say that $h_{i_{1}}$ and $h_{i_{2}}$ resolve $g_{i}$, we write $g_{i}=h_{i_{1}}+h_{i_{2}}$, and we call $h_{i_{1}}$ the complement of $h_{i_{2}}$ with respect to $g_{i}$, and vice versa. A row $g_{i}$ without 2 's is itself a haplotype and is uniquely resolved by this haplotype, which therefore has to be contained in $H$.

Problem: Parsimony Haplotyping $(P H)$

Input: A genotype matrix $G$.

Output: A haplotype matrix $H$ with a minimum number of rows that resolves $G$.

There is a rich literature in this area, of which recent papers such as [5] give a good overview. The problem is APX-hard [12][16] and the best known approximation algorithms are rather weak, yielding approximation guarantees of $2^{k-1}$ where $k$ is the maximum number of 2's appearing in a row of the genotype matrix [12][13]. The lack of success in finding strong approximation guarantees has led many authors to consider methods based on Integer Linear Programming (ILP) [5][10][11][12]. A different response to the hardness is to search for "islands of tractability" amongst special, restricted cases of the problem, exploring the frontier between hardness and polynomial-time solvability. In the literature available in this direction [6][13][16], this investigation has specified classes of $(k, l)$-bounded instances: in a $(k, l)$-bounded instance the input genotype matrix $G$ has at most $k 2$ 's per row and at most $l 2$ 's per column (cf. [16]). If $k$ or $l$ is a "*" we mean instances that are bounded only by the number of 2's per column or per row, respectively. This paper aims to supplement this "tractability" literature with mainly positive results, and doing so almost completes the bounded instance complexity landscape.

Next to the PH model we study a related model: the Minimum Perfect Phylogeny Haplotyping $(M P P H)$ model [2]. Again a minimum-size set of resolving haplotypes is required but this time under the additional, biologically-motivated restriction that the produced haplotypes permit a perfect phylogeny i.e. that they can be placed at the leaves of an evolutionary tree within which each site mutates at most once. Haplotype matrices admitting a perfect phylogeny are completely characterised [8][9] by the absence of the forbidden submatrix

$$
F=\left[\begin{array}{ll}
1 & 1 \\
0 & 0 \\
1 & 0 \\
0 & 1
\end{array}\right] .
$$


Problem: Minimum Perfect Phylogeny Haplotyping $(M P P H)$

Input: A genotype matrix $G$.

Output: A haplotype matrix $H$ with a minimum number of rows that resolves $G$ and admits a perfect phylogeny.

The feasibility question (PPH) - given a genotype matrix $G$, find any haplotype matrix $H$ that resolves $G$ and admits a perfect phylogeny, or state that no such $H$ exists - is solvable in linear-time [7][18]. Researchers in this area are now moving on to explore the PPH question on phylogenetic networks [17].

The $M P P H$ problem, however, has so far hardly been studied beyond an NPhardness result [2] and occasional comments within $P H$ and PPH literature [4][18][19]. In this paper we thus provide what is one of the first attempts to analyse the parsimony optimisation criteria within a well-defined and widely applicable biological framework. We seek namely to map the $M P P H$ complexity landscape in the same way as the $P H$ complexity landscape: using the concept of $(k, l)$-boundedness. We write $P H(k, l)$ and $M P P H(k, l)$ for these problems restricted to $(k, l)$-bounded instances.

In [12] it was shown that $P H(3, *)$ is APX-hard. In [6][13] it was shown that $P H(2, *)$ is polynomial-time solvable. Recently in [16], it was shown (amongst various other results) that $P H(4,3)$ is APX-hard. In this paper, we bring the boundaries between hard and easy classes closer by showing that $P H(3,3)$ is APX-hard and that $P H(*, 1)$ is polynomial-time solvable.

As far as $M P P H$ is concerned there have been, prior to this paper, no concrete results beyond the above mentioned NP-hardness result. We show that $M P P H(3,3)$ is APX-hard and that, like their $P H$ counterparts, $M P P H(2, *)$ and $M P P H(*, 1)$ are polynomial-time solvable (in both cases using a reduction to the $P H$ counterpart.)

For both problems the $(*, 2)$-bounded versions remain the intriguing open case. Analogous to a result from [16] for a subclass of $P H(*, 2)$, we show here that $M P P H(*, 2)$ is solvable in polynomial-time if the compatibility graph of the input genotype matrix is a clique. The compatibility graph $C(G)$ of a genotype matrix $G$ has vertices representing the rows (genotypes) of $G$, and there is an edge between two vertices if the corresponding two genotypes coincide in each column in which none of the two has a 2 . Our prediction is that learning the complexity of $P H(*, 2)$ and $M P P H(*, 2)$ in the case where the compatibility graph is a (graph-theoretical) sum of two or three cliques, will reveal the complexity of the full classes $P H(*, 2)$ and $M P P H(*, 2)$.

As explained by Sharan et al. in their "islands of tractability" paper [16], identifying tractable special classes can be practically useful for constructing highspeed subroutines within ILP solvers, but perhaps the most significant aspect of this paper is the analysis underpinning the results, which - by deepening our understanding of how this problem behaves - assists the search for better, faster approximation algorithms and for determining the exact beaches of the islands 
of tractability. Indeed, the continuing absence of approximation algorithms with strong accuracy guarantees underlines the importance of such work.

Furthermore, the fact that - prior to this paper - concrete and positive results for $M P P H$ had not been obtained (except for rather pessimistic modifications to ILP models [5]), means that the algorithms given here for the $M P P H$ cases, and the data structures used in their analysis (e.g. the restricted compatibility graph in Section 3), assume particular importance.

Finally, this paper yields some interesting open problems, of which the outstanding $(*, 2)$ case (for both $P H$ and $M P P H$ ) is only one; prominent amongst these questions (which are discussed at the end of the paper) is the question of whether $M P P H$ and $P H$ instances are inter-reducible, at least within the bounded-instance framework.

The paper is organised as follows. In Section 2 we give the hardness results, in Section 3 we present the polynomial-time solvable cases, and we finish in Section 4 with conclusions and open problems.

\section{Hard problems}

Theorem 1. $M P P H(3,3)$ is $A P X$-hard.

Proof. The proof in [2] that MPPH is NP-hard uses a reduction from VERTEX Cover, which can be modified to yield NP-hardness and APX-hardness for $(3,3)$-bounded instances. Given a graph $T=(V, E)$ the reduction in [2] constructs a genotype matrix $G(T)$ of $M P P H$ with $|V|+|E|$ rows and $2|V|+|E|$ columns. For every vertex $v_{i} \in V$ there is a genotype (row) $g_{i}$ in $G(T)$ with $g_{i}(i)=1, g_{i}(i+|V|)=1$ and $g_{i}(j)=0$ for every other position $j$. In addition, for every edge $e_{k}=\left\{v_{h}, v_{l}\right\}$ there is a genotype $g_{k}$ with $g_{k}(h)=2, g_{k}(l)=2$, $g_{k}(2|V|+k)=2$ and $g_{k}(j)=0$ for every other position $j$. Bafna et al. [2] prove that an optimal solution for $M P P H$ with input $G(T)$ contains $|V|+|E|+V C(T)$ haplotypes, where $V C(T)$ is the size of the smallest vertex cover in $T$.

3 -VERTEx COVER is the vertex cover problem when every vertex in the input graph has at most degree 3 . It is known to be APX-hard [14][1]. Let $T$ be an instance of 3-VERTEx COVER. We assume that $T$ is connected. Observe that for such a $T$ the reduction described above yields a $M P P H$ instance $G(T)$ that is $(3,3)$-bounded. We show that existence of a polynomial-time $(1+\epsilon)$ approximation algorithm $A(\epsilon)$ for $M P P H$ would imply a polynomial-time $\left(1+\epsilon^{\prime}\right)$ approximation algorithm for 3-VERTEX COVER with $\epsilon^{\prime}=8 \epsilon .^{1}$

Let $t$ be the solution value for $M P P H(G(T))$ returned by $A(\epsilon)$, and $t^{*}$ the optimal value for $M P P H(G(T))$. By the argument mentioned above from [2] we obtain a solution with value $d=t-|V|-|E|$ as an approximation of $V C(T)$.

\footnotetext{
${ }^{1}$ Strictly speaking this is insufficient to prove APX-hardness but it is not difficult to show that the described reduction is actually an L-reduction [14], from which APX-hardness follows.
} 
Since $t \leq(1+\epsilon) t^{*}$, we have $d \leq V C(T)+\epsilon V C(T)+\epsilon|V|+\epsilon|E|$. Connectedness of $T$ implies that $|V|-1 \leq|E|$. In 3-VERTEx COVER, a single vertex can cover at most 3 edges in $T$, implying that $V C(T) \geq|E| / 3 \geq(|V|-1) / 3$. Hence, $|V| \leq 4 V C(T)$ (for $|V| \geq 2$ ) and we have (if $|V| \geq 2$ ):

$$
\begin{aligned}
d & \leq V C(T)+\epsilon V C(T)+4 \epsilon V C(T)+3 \epsilon V C(T) \\
& \leq V C(T)+8 \epsilon V C(T) \\
& \leq(1+8 \epsilon) V C(T) .
\end{aligned}
$$

Theorem 2. $P H(3,3)$ is $A P X$-hard.

Proof. The proof by Sharan et al. [16] that $P H(4,3)$ is APX-hard can be modified slightly to obtain APX-hardness of $P H(3,3)$. The reduction is from 3Dimensional MATCHING with each element occurring in at most three triples (3DM3): given disjoint sets $X, Y$ and $Z$ containing $\nu$ elements each and a set $C=\left\{c_{0}, \ldots, c_{\mu-1}\right\}$ of $\mu$ triples in $X \times Y \times Z$ such that each element occurs in at most three triples in $C$, find a maximum cardinality set $C^{\prime} \subseteq C$ of disjoint triples.

From an instance of 3DM3 we build a genotype matrix $G$ with $3 \nu+3 \mu$ rows and $6 \nu+4 \mu$ columns. The first $3 \nu$ rows are called element-genotypes and the last $3 \mu$ rows are called matching-genotypes. We specify non-zero entries of the genotypes only. ${ }^{2}$ For every element $x_{i} \in X$ define element-genotype $g_{i}^{x}$ with $g_{i}^{x}(3 \nu+i)=1 ; g_{i}^{x}(6 \nu+4 k)=2$ for all $k$ with $x_{i} \in c_{k}$. If $x_{i}$ occurs in at most two triples we set $g_{i}^{x}(i)=2$. For every element $y_{i} \in Y$ there is an element-genotype $g_{i}^{y}$ with $g_{i}^{y}(4 \nu+i)=1 ; g_{i}^{y}(6 \nu+4 k)=2$ for all $k$ with $y_{i} \in c_{k}$ and if $y_{i}$ occurs in at most two triples then we set $g_{i}^{y}(\nu+i)=2$. For every element $z_{i} \in Z$ there is an element-genotype $g_{i}^{z}$ with $g_{i}^{z}(5 \nu+i)=1 ; g_{i}^{z}(6 \nu+4 k)=2$ for all $k$ with $z_{i} \in c_{k}$ and if $z_{i}$ occurs in at most two triples then we set $g_{i}^{z}(2 \nu+i)=2$. For each triple $c_{k}=\left\{x_{i_{1}}, y_{i_{2}}, z_{i_{3}}\right\} \in C$ there are three matching-genotypes $c_{k}^{x}, c_{k}^{y}$ and $c_{k}^{z}: c_{k}^{x}$ has $c_{k}^{x}\left(3 \nu+i_{1}\right)=2, c_{k}^{x}(6 \nu+4 k)=1$ and $c_{k}^{x}(6 \nu+4 k+1)=2 ; c_{k}^{y}$ has $c_{k}^{y}\left(4 \nu+i_{2}\right)=2, c_{k}^{y}(6 \nu+4 k)=1$ and $c_{k}^{y}(6 \nu+4 k+2)=2 ; c_{k}^{z}$ has $c_{k}^{z}\left(5 \nu+i_{3}\right)=2$, $c_{k}^{z}(6 \nu+4 k)=1$ and $c_{k}^{z}(6 \nu+4 k+3)=2$.

Notice that the element-genotypes only have a 2 in the first $3 \nu$ columns if the element occurs in at most two triples. This is the only difference with the reduction from [16], where every element-genotype has a 2 in the first $3 \nu$ columns: i.e., for elements $x_{i} \in X, y_{i} \in Y$ or $z_{i} \in Z$ a 2 in column $i, \nu+i$ or $2 \nu+i$, respectively. As a direct consequence our genotype matrix has only three 2's per row in contrast to the four 2's per row in the original reduction.

We claim that for this (3,3)-bounded instance exactly the same arguments can be used as for the (4,3)-bounded instance. In the original reduction the leftmost 2's ensured that, for each element-genotype, at most one of the two haplotypes used to resolve it was used in the resolution of other genotypes. Clearly this remains true in our modified reduction for elements appearing in two or fewer

\footnotetext{
${ }^{2}$ Only in this proof we index haplotypes, genotypes and matrices starting with 0 ,
} which makes notation consistent with [16]. 
triples, because the corresponding left-most 2's have been retained. So consider an element $x_{i}$ appearing in three triples and suppose, by way of contradiction, that both haplotypes used to resolve $g_{i}^{x}$ are used in the resolution of other genotypes. Now, the 1 in position $3 \nu+i$ prevents this element-genotype from sharing haplotypes with other element-genotypes, so genotype $g_{i}^{x}$ must share both its haplotypes with matching-genotypes. Note that, because $g_{i}^{x}(3 \nu+i)=1$, the genotype $g_{i}^{x}$ can only possibly share haplotypes with matching-genotypes corresponding to triples that contain $x_{i}$. Indeed, if $x_{i}$ is in triples $c_{k_{1}}, c_{k_{2}}$ and $c_{k_{3}}$ then the only genotypes with which $g_{i}^{x}$ can potentially share haplotypes are $c_{k_{1}}^{x}, c_{k_{2}}^{x}$ and $c_{k_{3}}^{x}$. Genotype $g_{i}^{x}$ cannot share both its haplotypes with the same matchinggenotype (e.g. $c_{k_{1}}^{x}$ ) because both haplotypes of $g_{i}^{x}$ will have a 1 in column $3 \nu+i$ whilst only one of the two haplotypes for $c_{k_{1}}^{x}$ will have a 1 in that column. So, without loss of generality, $g_{i}^{x}$ is resolved by a haplotype that $c_{k_{1}}^{x}$ uses and a haplotype that $c_{k_{2}}^{x}$ uses. However, this is not possible, because $g_{i}^{x}$ has a 2 in the column corresponding to $c_{k_{3}}$, whilst both $c_{k_{1}}^{x}$ and $c_{k_{2}}^{x}$ have a 0 in that column, yielding a contradiction.

Note that, in the original reduction, it was not only true that each elementgenotype shared at most one of its haplotypes, but - more strongly - it was also true that such a shared haplotype was used by exactly one other genotype (i.e. the genotype corresponding to the triple the element gets assigned to). To see that this property is also retained in the modified reduction observe that if (say) $g_{i}^{x}$ shares one haplotype with two genotypes $c_{k_{1}}^{x}$ and $c_{k_{2}}^{x}$ then $x_{i}$ must be in both triples $c_{k_{1}}$ and $c_{k_{2}}$, but this is not possible because, in the two columns corresponding to triples $c_{k_{1}}$ and $c_{k_{2}}, c_{k_{1}}^{x}$ has 1 and 0 whilst $c_{k_{2}}^{x}$ has 0 and 1 .

\section{Polynomial-time solvability}

\subsection{Parsimony haplotyping}

The following result shows the polynomial-time solvability of $P H$ on $(*, 1)$ bounded instances.

We say that two genotypes $g_{1}$ and $g_{2}$ are compatible, denoted as $g_{1} \sim g_{2}$, if $g_{1}(j)=g_{2}(j)$ or $g_{1}(j)=2$ or $g_{2}(j)=2$ for all $j$. A genotype $g$ and a haplotype $h$ are consistent if $h$ can be used to resolve $g$, ie. if $g(j)=h(j)$ or $g(j)=2$ for all $j$. The compatibility graph is the graph with vertices for the genotypes and an edge between two genotypes if they are compatible.

Lemma 1. If $g_{1}$ and $g_{2}$ are rows of a genotype matrix with at most one 2 per column and $g_{1}$ and $g_{2}$ are compatible then there exists exactly one haplotype that is consistent with both $g_{1}$ and $g_{2}$.

Proof. The only haplotype that is consistent with both $g_{1}$ and $g_{2}$ is $h$ with $h(j)=g_{1}(j)$ for all $j$ with $g_{1}(j) \neq 2$ and $h(j)=g_{2}(j)$ for all $j$ with $g_{2}(j) \neq 2$. There are no columns where $g_{1}$ and $g_{2}$ are both equal to 2 because there is at most one 2 per column. In columns where $g_{1}$ and $g_{2}$ are both not equal to 2 they are equal because $g_{1}$ and $g_{2}$ are compatible. 
We use the notation $g_{1} \sim_{h} g_{2}$ if $g_{1}$ and $g_{2}$ are compatible and $h$ is consistent with both. We prove that the compatibility graph has a specific structure. A 1-sum of two graphs is the result of identifying a vertex of one graph with a vertex of the other graph. A 1-sum of $n+1$ graphs is the result of identifying a vertex of a graph with a vertex of a 1-sum of $n$ graphs. See Figure 1 for an example of a 1-sum of three cliques $\left(K_{3}, K_{4}\right.$ and $\left.K_{2}\right)$.

Lemma 2. If $G$ is a genotype matrix with at most one 2 per column then every connected component of the compatibility graph of $G$ is a 1-sum of cliques, where edges in the same clique are labelled with the same haplotype.

Proof. Let $C$ be the compatibility graph of $G$ and let $g_{1}, g_{2}, \ldots, g_{k}$ be a cycle in $C$. It suffices to show that there exists a haplotype $h_{c}$ such that $g_{i} \sim_{h_{c}} g_{i^{\prime}}$ for all $i, i^{\prime} \in\{1, \ldots, k\}$. Consider an arbitrary column $j$. If there is no genotype with a 2 in this column then $g_{1} \sim g_{2} \sim \ldots \sim g_{k}$ implies that $g_{1}(j)=g_{2}(j)=\ldots=$ $g_{k}(j)$. Otherwise, let $g_{i_{j}}$ be the unique genotype with a 2 in column $j$. Then $g_{1} \sim g_{2} \sim \ldots \sim g_{i_{j}-1}$ together with $g_{1} \sim g_{k} \sim g_{k-1} \sim \ldots \sim g_{i_{j}+1}$ implies that $g_{i}(j)=g_{i^{\prime}}(j)$ for all $i, i^{\prime} \in\{1, \ldots, k\} \backslash\left\{i_{j}\right\}$. Set $h_{c}(j)=g_{i}(j), i \neq i_{j}$. Repeating this for each column $j$ produces a haplotype $h_{c}$ such that indeed $g_{i} \sim_{h_{c}} g_{i^{\prime}}$ for all $i, i^{\prime} \in\{1, \ldots, k\}$.

$g_{1}$
$g_{2}$
$g_{3}$
$g_{4}$
$g_{5}$
$g_{6}$
$g_{7}$$\left[\begin{array}{lllllllllll}0 & 0 & 1 & 0 & 2 & 0 & 1 \\ 2 & 0 & 2 & 0 & 0 & 0 & 1 \\ 0 & 0 & 1 & 1 & 0 & 0 & 0 & 0 & 0 & 2 \\ 0 & 0 & 1 & 1 & 0 & 2 & 1 \\ 1 & 2 & 0 & 0 & 0 & 0 & 1 \\ 0 & 0 & 1 & 1 & 0 & 0 & 1\end{array}\right]$

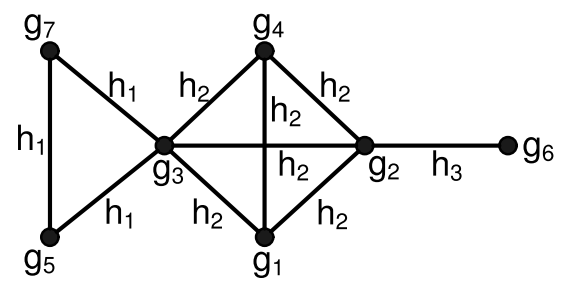

Figure 1. Example of a genotype matrix and the corresponding compatibility graph, with $h_{1}=(0,0,1,1,0,0,1), h_{2}=(0,0,1,0,0,0,1)$ and $h_{3}=(1,0,0,0,0,0,1)$.

From this lemma, it follows directly that in $P H(*, 1)$ the compatibility graph is chordal, meaning that all its induced cycles are triangles. Every chordal graph has a simplicial vertex, a vertex whose (closed) neighbourhood is a clique. Deleting a vertex in a chordal graph gives again a chordal graph (see for example [3] for an introduction to chordal graphs). The following lemma leads almost immediately to polynomial solvability of $P H(*, 1)$. We use set-operations for the rows of matrices: thus, e.g., $h \in H$ says $h$ is a row of matrix $H, H \cup h$ says $h$ is added to $H$ as a row, and $H^{\prime} \subset H$ says $H^{\prime}$ is a submatrix consisting of rows of $H$.

Lemma 3. Given haplotype matrix $H^{\prime}$ and genotype matrix $G$ with at most one 2 per column it is possible to find, in polynomial time, a haplotype matrix $H$ that resolves $G$, has $H^{\prime}$ as a submatrix and has a minimum number of rows.

Proof. The proof is constructive. Let problem $\left(G, H^{\prime}\right)$ denote the above problem on input matrices $G$ and $H^{\prime}$. Let $C$ be the compatibility graph of $G$, which implied by Lemma 2 is chordal. Suppose $g$ corresponds to a simplicial vertex of $C$. Let $h_{c}$ be the unique haplotype consistent with any genotype in the closed neighbourhood clique of $g$. We extend matrix $H^{\prime}$ to $H^{\prime \prime}$ and update graph $C$ as follows. 
1. If $g$ has no 2's it can be resolved with only one haplotype $h=g$. We set $H^{\prime \prime}=H^{\prime} \cup h$ and remove $g$ from $C$.

2. Else, if there exist rows $h_{1} \in H^{\prime}$ and $h_{2} \in H^{\prime}$ that resolve $g$ we set $H^{\prime \prime}=H^{\prime}$ and remove $g$ from $C$.

3. Else, if there exists $h_{1} \in H^{\prime}$ such that $g=h_{1}+h_{c}$ we set $H^{\prime \prime}=H^{\prime} \cup h_{c}$ and remove $g$ from $C$.

4. Else, if there exists $h_{1} \in H^{\prime}$ and $h_{2} \notin H^{\prime}$ such that $g=h_{1}+h_{2}$ we set $H^{\prime \prime}=H^{\prime} \cup h_{2}$ and remove $g$ from $C$.

5. Else, if $g$ is not an isolated vertex in $C$ then there exists a haplotype $h_{1}$ such that $g=h_{1}+h_{c}$ and we set $H^{\prime \prime}=H^{\prime} \cup\left\{h_{1}, h_{c}\right\}$ and remove $g$ from $C$.

6. Otherwise, $g$ is an isolated vertex in $C$ and we set $H^{\prime \prime}=H^{\prime} \cup\left\{h_{1}, h_{2}\right\}$ for any $h_{1}$ and $h_{2}$ such that $g=h_{1}+h_{2}$ and remove $g$ from $C$.

The resulting graph is again chordal and we repeat the above procedure for $H^{\prime}=H^{\prime \prime}$ until all vertices are removed from $C$. Let $H$ be the final haplotype matrix $H^{\prime \prime}$. It is clear from the construction that $H$ resolves $G$.

We prove that $H$ has a minimum number of rows by induction on the number of genotypes. Clearly, if $G$ has only one genotype the algorithm constructs the only, and hence optimal, solution. The induction hypothesis is that the algorithm finds an optimal solution to the problem $\left(G, H^{\prime}\right)$ for any haplotype matrix $H^{\prime}$ if $G$ has at most $n-1$ rows. Now consider haplotype matrix $H^{\prime}$ and genotype matrix $G$ with $n$ rows. The first step of the algorithm selects a simplicial vertex $g$ and proceeds with one of the cases 1 to 6 . The algorithm then finds (by the induction hypothesis) an optimal solution $H$ to problem $\left(G \backslash\{g\}, H^{\prime \prime}\right)$. It remains to prove that $H$ is also an optimal solution to problem $\left(G, H^{\prime}\right)$. We do this by showing that an optimal solution $H^{*}$ to problem $\left(G, H^{\prime}\right)$ can be modified to include $H^{\prime \prime}$. We prove this for every case of the algorithm separately.

1. In this case $h \in H^{*}$, since $g$ can only be resolved by $h$.

2. In this case $H^{\prime \prime}=H^{\prime}$ and hence $H^{\prime \prime} \subseteq H^{*}$.

3. Suppose that $h_{c} \notin H^{*}$. Because we are not in case 2 we know that there are two rows in $H^{*}$ that resolve $g$ and at least one of the two, say $h^{*}$, is not a row of $H^{\prime}$. Since $h_{c}$ is the unique haplotype consistent with (the simplicial) $g$ and any compatible genotype, $h^{*}$ can not be consistent with any other genotype than $g$. Thus, replacing $h^{*}$ by $h_{c}$ gives a solution with the same number of rows but containing $h_{c}$.

4. Suppose that $h_{2} \notin H^{*}$. Because we are not in case 2 or 3 we know that there is a haplotype $h^{*} \in H^{*}$ consistent with $g, h^{*} \notin H^{\prime}$ and $h^{*} \neq h_{c}$. Hence it is not consistent with any other genotypes than $g$ and we can replace $h^{*}$ by $h_{2}$.

5. Suppose that $h_{1} \notin H^{*}$ or $h_{c} \notin H^{*}$. Because we are not in case 2,3 or 4 , there are haplotypes $h^{*} \in H \backslash H^{\prime}$ and $h^{* *} \in H \backslash H^{\prime}$ that resolve $g$. If $h^{*}$ and $h^{* *}$ are both not equal to $h_{c}$ then they are not consistent with any other genotype than $g$. Replacing $h^{*}$ and $h^{* *}$ by $h_{1}$ and $h_{c}$ leads to another optimal solution. If one of $h^{*}$ and $h^{* *}$ is equal to $h_{c}$ then we can replace the other one by $h_{1}$. 
6. Suppose that $h_{1} \notin H^{*}$ or $h_{2} \notin H^{*}$. There are haplotypes $h^{*}, h^{* *} \in H^{*} \backslash H^{\prime}$ that resolve $g$ and just $g$ since $g$ is an isolated vertex. Replacing $h^{*}$ and $h^{* *}$ by $h_{1}$ and $h_{2}$ gives an optimal solution containing $h_{1}$ and $h_{2}$.

Theorem 3. The problem $P H(*, 1)$ can be solved in polynomial time.

Proof. The proof follows from Lemma 3. Construction of the compatibility graph takes $O\left(n^{2} m\right)$ time, for an $n$ times $m$ input matrix. Finding an ordering in which to delete the simplicial vertices can be done in time $O\left(n^{2}\right)$ (see [15]) and resolving each vertex takes $O\left(n^{2} m\right)$ time. The overall running time of the algorithm is therefore $O\left(n^{3} m\right)$.

\subsection{Minimum pure parsimony haplotyping}

Polynomial-time solvability of $P H$ on $(2, *)$-bounded instances has been shown in [6] and [13]. We prove it for $\operatorname{MPPH}(2, *)$. We start with a definition.

Definition 1. For two columns of a genotype matrix we say that a reduced resolution of these columns is the result of applying the following rules as often as possible to the submatrix induced by these columns: deleting one of two identical rows and the replacement rules

$$
\left[\begin{array}{ll}
2 & a
\end{array}\right] \rightarrow\left[\begin{array}{ll}
1 & a \\
0 & a
\end{array}\right],\left[\begin{array}{ll}
a & 2
\end{array}\right] \rightarrow\left[\begin{array}{ll}
a & 1 \\
a & 0
\end{array}\right],\left[\begin{array}{ll}
2 & 2
\end{array}\right] \rightarrow\left[\begin{array}{ll}
1 & 1 \\
0 & 0
\end{array}\right] \text { and }\left[\begin{array}{ll}
2 & 2
\end{array}\right] \rightarrow\left[\begin{array}{ll}
1 & 0 \\
0 & 1
\end{array}\right], \text { for } a \in\{0,1\}
$$

Note that two columns can have more than one reduced resolution if there is a genotype with a 2 in both these columns. The reduced resolutions of a column pair of a genotype matrix $G$ are submatrices of (or equal to) $F$ and represent all possibilities for the submatrix induced by the corresponding two columns of a minimal haplotype matrix $H$ resolving $G$, after collapsing identical rows.

Theorem 4. The problem MPPH $(2, *)$ can be solved in polynomial time.

Proof. We reduce $M P P H(2, *)$ to $P H\left(2,{ }^{*}\right)$, which can be solved in polynomial time (see above). Let $G$ be an instance of $M P P H(2, *)$. We may assume that any two rows are different.

Take the submatrix of any two columns of $G$. If it does not contain a [ $\left.\begin{array}{ll}2 & 2\end{array}\right]$ row, then in terms of Definition 1 there is only one reduced resolution. If $G$ contains two or more [2 2 ] rows then, since by assumption all genotypes are different, $G$ must have $\left[\begin{array}{lll}2 & 2 & 0 \\ 2 & 2 & 1\end{array}\right]$ and therefore $\left[\begin{array}{ll}2 & 0 \\ 2 & 1\end{array}\right]$ as a submatrix, which can only be resolved by a haplotype matrix containing the forbidden submatrix $F$. It follows that in this case the instance is infeasible. If it contains exactly one $\left[\begin{array}{ll}2 & 2\end{array}\right]$ row, then there are clearly two reduced resolutions. Thus we may assume that for each column pair there are at most two reduced solutions.

Observe that if for some column pair all reduced resolutions are equal to $F$ the instance is again infeasible. On the other hand, if for all column pairs none of the reduced resolutions is equal to $F$ then $\operatorname{MPPH}(2, *)$ is equivalent 
to $P H(2, *)$ because any minimal haplotype matrix $H$ that resolves $G$ admits a perfect phylogeny. Finally, consider a column pair with two reduced resolutions, one of them containing $F$. Because there are two reduced resolutions there is a genotype $g$ with a 2 in both columns. Let $h_{1}$ and $h_{2}$ be the haplotypes that correspond to the resolution of $g$ that does not lead to $F$. Then we replace $g$ in $G$ by $h_{1}$ and $h_{2}$, ensuring that a minimal haplotype matrix $H$ resolving $G$ can not have $F$ as a submatrix in these two columns.

Repeating this procedure for every column pair either tells us that the matrix $G$ was an infeasible instance or creates a genotype matrix $G^{\prime}$ such that any minimal haplotype matrix $H$ resolves $G^{\prime}$ if and only if $H$ resolves $G$, and $H$ admits a perfect phylogeny.

Theorem 5. The problem MPPH $(*, 1)$ can be solved in polynomial time.

Proof. Similar to the proof of Theorem 4 we reduce $\operatorname{MPPH}(*, 1)$ to $\operatorname{PH}(*, 1)$. As there, consider for any pair of columns of the input genotype matrix $G$ its reduced resolutions, according to Definition 1 . Since $G$ has at most one 2 per column there is at most one genotype with 2's in both columns. Hence there are at most two reduced resolutions. If all reduced resolutions are equal to the forbidden submatrix $F$ the instance is infeasible. If on the other hand for all column pairs no reduced resolution is equal to $F$ then in fact $\operatorname{MPPH}(*, 1)$ is equivalent to $P H(*, 1)$, because any minimal haplotype matrix resolving $G$ admits a perfect phylogeny.

As in the proof of Theorem 4 we are left with considering column pairs for which one of the two reduced resolutions is equal to $F$. For such a column pair there must be a genotype $g$ that has 2's in both these columns. The other genotypes have only 0's and 1's in them. Suppose we get a forbidden submatrix $F$ in these columns of the solution if $g$ is resolved by haplotypes $h_{1}$ and $h_{2}$, where $h_{1}$ has $a$ and $b$ and therefore $h_{2}$ has $1-a$ and $1-b$ in these columns, $a, b \in\{0,1\}$. We will change the input matrix $G$ such that if $g$ gets resolved by such a forbidden resolution these haplotypes are not consistent with any other genotypes. We do this by adding an extra column to $G$ as follows. The genotype $g$ gets a 1 in this new column. Every genotype with $a$ and $b$ or with $1-a$ and $1-b$ in the considered columns gets a 0 in the new column. Every other genotype gets a 1 in the new column. For example, the matrix

$$
\left[\begin{array}{ll}
2 & 2 \\
0 & 1 \\
1 & 0 \\
1 & 1
\end{array}\right] \text { gets one extra column and becomes }\left[\begin{array}{lll}
2 & 2 & 1 \\
0 & 1 & 1 \\
1 & 0 & 1 \\
1 & 1 & 0
\end{array}\right] \text {. }
$$

Denote by $G_{\text {mod }}$ the result of modifying $G$ by adding such a column for every pair of columns with exactly one 'bad' and one 'good' reduced resolution. It is not hard to see that any optimal solution to $P H(*, 1)$ on $G_{m o d}$ can be transformed into a solution to $M P P H(*, 1)$ on $G$ of the same cardinality (indeed, any two haplotypes used in a forbidden resolution of a genotype $g$ in $G_{\text {mod }}$ are not consistent with any other genotype of $G_{m o d}$, and hence may be replaced by 
two other haplotypes resolving $g$ in a non-forbidden way). Now, let $H$ be an optimal solution to $\operatorname{MPPH}(*, 1)$ on $G$. We can modify $H$ to obtain a solution to $P H(*, 1)$ on $G_{m o d}$ of the same cardinality as follows. We modify every haplotype in $H$ in the same way as the genotypes it resolves. From the construction of $G_{m o d}$ it follows that two compatible genotypes are only modified differently if the haplotype they are both consistent with is in a forbidden resolution. However, in $H$ no genotypes are resolved with a forbidden resolution since $H$ is a solution to $M P P H(*, 1)$. We conclude that optimal solutions to $P H(*, 1)$ on $G_{m o d}$ correspond to optimal solutions to $\operatorname{MPPH}(*, 1)$ on $G$ and hence the latter problem can be solved in polynomial time, by Theorem 3 .

If we use the algorithm from the proof of Lemma 3 as a subroutine we get an overall running time of $O\left(n^{3} m^{2}\right)$, for an $n \times m$ input matrix.

The borderline open complexity problems in $P H$ and $M P P H$ are now $P H(*, 2)$ and $M P P H(*, 2)$. Unfortunately, we have not found the answer to these complexity questions. However, the borders have been pushed slightly further. In [16] $\mathrm{PH}(*, 2)$ is shown to be polynomially solvable if the input genotypes have the complete graph as compatibility graph, we call this problem $P H(*, 2)-C 1$. We will give the counterpart result for $M P P H(*, 2)-C 1$.

Let $G$ be an $n \times m M P P H(*, 2)-C 1$ input matrix. Since the compatibility graph is a clique, every column of $G$ contains only one symbol besides possible 2 's. If we replace in every 1-column of $G$ (a column containing only 1's and 2's) the 1's by 0's and mark the SNP corresponding to this column 'flipped', then we obtain an equivalent problem on a $\{0,2\}$-matrix $G^{\prime}$. To see that this problem is indeed equivalent, suppose $H^{\prime}$ is a haplotype matrix resolving this modified genotype matrix $G^{\prime}$ and suppose $H^{\prime}$ does not contain the forbidden submatrix $F$. Then by interchanging 0 's and 1's in every column of $H^{\prime}$ corresponding to a flipped SNP, one obtains a haplotype matrix $H$ without the forbidden submatrix which resolves the original input matrix $G$. And vice versa. Hence, from now on we will assume, without loss of generality, that the input matrix $G$ is a $\{0,2\}$ matrix.

If we assume moreover that $n \geq 3$, which we do from here on, the trivial haplotype $h_{t}$ defined as the all-0 haplotype of length $m$ is the only haplotype consistent with all genotypes in $G$.

We define the restricted compatibility graph $C_{R}(G)$ of $G$ as follows. As in the normal compatibility graph, the vertices of $C_{R}(G)$ are the genotypes of $G$. However, there is an edge $\left\{g, g^{\prime}\right\}$ in $C_{R}(\mathrm{G})$ only if $g \sim_{h} g^{\prime}$ for some $h \neq h_{t}$, or, equivalently, if there is a column where both $g$ and $g^{\prime}$ have a 2 .

Lemma 4. If $G$ is a feasible instance of $M P P H(*, 2)-C 1$ then every vertex in $C_{R}(G)$ has degree at most 2.

Proof. Any vertex of degree higher than 2 in $C_{R}(G)$ implies the existence in $G$ of submatrix: 


$$
B=\left[\begin{array}{lll}
2 & 2 & 2 \\
2 & 0 & 0 \\
0 & 2 & 0 \\
0 & 0 & 2
\end{array}\right]
$$

It is easy to verify that no resolution of this submatrix permits a perfect phylogeny.

Suppose that $G$ has two identical columns. There are either 0,1 or 2 rows with 2 's in both these columns. In each case it is easy to see that any haplotype matrix $H$ resolving $G$ can be modified, without introducing a forbidden submatrix, to make the corresponding columns in $H$ equal as well (simply delete one column and duplicate another). This leads to the first step of the algorithm $\mathbf{A}$ that we propose for solving $M P P H(*, 2)-C 1$ :

Step 1 of A: Collapse all identical columns in $G$.

From now on, we assume that there are no identical columns. Let us partition the genotypes in $G_{0}, G_{1}$ and $G_{2}$, denoting the set of genotypes in $G$ with, respectively, degree 0,1 , and 2 in $C_{R}(G)$. For any genotype $g$ of degree 1 in $C_{R}(G)$ there is exactly one genotype with a 2 in the same column as $g$. Because there are no identical columns, it follows that any genotype $g$ of degree 1 in $C_{R}(G)$ can have at most two 2's. Similarly any genotype of degree 2 in $C_{R}(G)$ has at most three 2's. Accordingly we define $G_{1}^{1}$ and $G_{1}^{2}$ as the genotypes in $G_{1}$ that have one 2 and two 2's, respectively, and similarly $G_{2}^{2}$ and $G_{2}^{3}$ as the genotypes in $G_{2}$ with two and three 2's, respectively.

The following lemma states how genotypes in these sets must be resolved if no submatrix $F$ is allowed in the solution. If genotype $g$ has $k 2$ 's we denote by $g\left[a_{1}, a_{2}, \ldots, a_{k}\right]$ the haplotype with entry $a_{i}$ in the position where $g$ has its $i$-th 2 and 0 everywhere else.

Lemma 5. In a feasible solution to the problem $M P P H(*, 2)-C 1$ all genotypes are resolved in one of the following ways:

(i) A genotype $g \in G_{1}^{1}$ is resolved by $g[1]$ and $g[0]=h_{t}$.

(ii) A genotype $g \in G_{2}^{2}$ is resolved by $g[0,1]$ and $g[1,0]$.

(iii) A genotype $g \in G_{1}^{2}$ is either resolved by $g[0,0]=h_{t}$ and $g[1,1]$ or by $g[0,1]$ and $g[1,0]$.

(iv) A genotype $g \in G_{2}^{3}$ is either resolved by $g[1,0,0]$ and $g[0,1,1]$ or by $g[0,1,0]$ and $g[1,0,1]$ (assuming that the two neighbours of $g$ have $a 2$ in the first two positions where $g$ has a 2).

Proof. A genotype $g \in G_{2}^{2}$ has degree 2 in $C_{R}(G)$, which implies the existence in $G$ of a submatrix:

$$
D=\begin{aligned}
& g \\
& g^{\prime} \\
& g^{\prime \prime}
\end{aligned}\left[\begin{array}{ll}
2 & 2 \\
2 & 0 \\
0 & 2
\end{array}\right] \text {. }
$$

Resolving $g$ with $g[0,0]$ and $g[1,1]$ clearly leads to the forbidden submatrix $F$. Similarly, resolving a genotype $g \in G_{2}^{3}$ with $g[0,0,1]$ and $g[1,1,0]$ or with $g[0,0,0]$ 
and $g[1,1,1]$ leads to a forbidden submatrix in the first two columns where $g$ has a 2. It follows that resolving the genotypes in a way other than described in the lemma yields a haplotype matrix which does not admit a perfect phylogeny.

Now suppose that all genotypes are resolved as described in the lemma and assume that there is a forbidden submatrix $F$ in the solution. Without loss of generality, we assume $F$ can be found in the first two columns of the solution matrix. We may also assume that no haplotype can be deleted from the solution. Then, since $F$ contains [1 1 ], there is a genotype $g$ starting with [2 2]. Since there are no identical columns there are only two possibilities. The first possibility is that there is exactly one other genotype $g^{\prime}$ with a 2 in exactly one of the first two columns. Since all genotypes different from $g$ and $g^{\prime}$ start with $[0$ 0], none of the resolutions of $g$ can have created the complete submatrix $F$. Contradiction. The other possibility is that there is exactly one genotype with a 2 in the first column and exactly one genotype with a 2 in the second column, but these are different genotypes, i.e. we have the submatrix $D$. Then $g \in G_{2}^{3}$ or $g \in G_{2}^{2}$ and it can again be checked that none of the resolutions in (ii) and (iv) leads to the forbidden submatrix.

Lemma 6. Let $G$ be an instance of $M P P H(*, 2)$ and $G_{1}^{2}, G_{2}^{3}$ as defined above. (i) Any nontrivial haplotype is consistent with at most two genotypes in $G$.

(ii) A genotype $g \in G_{1}^{2} \cup G_{2}^{3}$ must be resolved using at least one haplotype that is not consistent with any other genotype.

Proof. (i) Let $h$ be a nontrivial haplotype. There is a column where $h$ has a 1 and there are at most two genotypes with a 2 in that column.

(ii) A genotype $g \in G_{1}^{2} \cup G_{2}^{3}$ has a 2 in a column that has no other 2's. Hence there is a haplotype with a 1 in this column and this haplotype is not consistent with any other genotypes.

A haplotype that is only consistent with $g$ is called a private haplotype of $g$. Based on (i) and (ii) of Lemma 5 we propose the next step of $\mathbf{A}$ :

Step 2 of A: Resolve all $g \in G_{1}^{1} \cup G_{2}^{2}$ by the unique haplotypes allowed to resolve them according to Lemma 5. Also resolve each $g \in G_{0}$ with $h_{t}$ and the complement of $h_{t}$ with respect to $g$. This leads to a partial haplotype matrix $H_{2}^{p}$.

The next step of $\mathbf{A}$ is based on Lemma 6 (ii).

Step 3 of A: For each $g \in G_{1}^{2} \cup G_{2}^{3}$ with $g \sim_{h^{\prime}} g^{\prime}$ for some $h^{\prime} \in H_{2}^{p}$ that is allowed to resolve $g$ according to Lemma 5 , resolve $g$ by adding the complement $h^{\prime \prime}$ of $h^{\prime}$ w.r.t. $g$ to the set of haplotypes, i.e. set $H_{2}^{p}:=H_{2}^{p} \cup\left\{h^{\prime \prime}\right\}$, and repeat this step as long as new haplotypes get added. This leads to partial haplotype matrix $H_{3}^{p}$.

Notice that $H_{3}^{p}$ does not contain any haplotype that is allowed to resolve any of the genotypes that have not been resolved in Steps 2 and 3. Let us denote this set of leftover, unresolved haplotypes by $G L$, the degree 1 vertices among those by $G L_{1} \subseteq G_{1}^{2}$, and the degree 2 vertices among those by $G L_{2} \subseteq G_{2}^{3}$. The restricted compatibility graph induced by $G L$, which we denote by $C_{R}(G L)$ 
consists of paths and circuits. We first give the final steps of algorithm A and argue optimality afterwards.

Step 4 of A: Resolve each cycle in $C_{R}(G L)$, necessarily consisting of $G L_{2^{-}}$ vertices, by starting with an arbitrary vertex and, following the cycle, resolving each next pair $g, g^{\prime}$ of vertices by haplotype $h \neq h_{t}$ such that $g \sim_{h} g^{\prime}$ and the two complements of $h$ w.r.t. $g$ and $g^{\prime}$ respectively. In case of an odd cycle the last vertex is resolved by any pair of haplotypes that is allowed to resolve it. Note that $h$ has a 1 in the column where both $g$ and $g^{\prime}$ have a 2 and otherwise 0 . It follows easily that $g$ and $g^{\prime}$ are both allowed to use $h$ (and its complement) according to (iv) of Lemma 5 .

Step 5 of A: Resolve each path in $C_{R}(G L)$ with both endpoints in $G L_{1}$ by first resolving the $G L_{1}$ endpoints by the trivial haplotype $h_{t}$ and the complements of $h_{t}$ w.r.t. the two endpoint genotypes, respectively. The remaining path contains only $G L_{2}$-vertices and is resolved according to Step 6 .

Step 6 of A: Resolve each remaining path by starting in (one of) its $G L_{2^{-}}$ endpoint(s), and following the path, resolving each next pair of vertices as in Step 4. In case of a path with an odd number of vertices, resolve the last vertex by any pair of haplotypes that is allowed to resolve it in case it is a $G L_{2}$-vertex, and resolve it by the trivial haplotype and its complement w.r.t. the vertex in case it is a $G L_{1}$ vertex.

By construction the haplotype matrix $H$ resulting from $\mathbf{A}$ resolves $G$. In addition, from Lemma 5 follows that $H$ admits a perfect phylogeny.

To argue minimality of the solution, first observe that the haplotypes added in Step 2 and Step 3 are unavoidable by Lemma 5 (i) and (ii) and Lemma 6 (ii). Lemma 6 tells us moreover that the resolution of a cycle of $k$ genotypes in $G L_{2}$ requires at least $k+\left\lceil\frac{k}{2}\right\rceil$ haplotypes that can not be used to resolve any other genotypes in $G L$. This proves optimality of Step 4. To prove optimality of the last two steps we need to take into account that genotypes in $G L_{1}$ can potentially share the trivial haplotype. Observe that to resolve a path with $k$ vertices one needs at least $k+\left\lceil\frac{k}{2}\right\rceil$ haplotypes. Indeed $\mathbf{A}$ does not use more than that in Steps 5 and 6. Moreover, since these paths are disjoint, they cannot share haplotypes for resolving their genotypes except for the endpoints if they are in $G L_{1}$, which can share the trivial haplotype. Indeed, $\mathbf{A}$ exploits the possibility of sharing the trivial haplotype in a maximal way, except on a path with an even number of vertices and one endpoint in $G L_{1}$. Such a path, with $k$ (even) vertices, is resolved in $\mathbf{A}$ by $3 \frac{k}{2}$ haplotypes that can not be used to resolve any other genotypes. The degree 1 endpoint might alternatively be resolved by the trivial haplotype and its complement w.r.t. the corresponding genotype, adding the latter private haplotype, but then for resolving the remaining path with $k-1$ (odd) vertices only from $G L_{2}$ we still need $k-1+\left\lceil\frac{k-1}{2}\right\rceil$, which together with the private haplotype of the degree 1 vertex gives $3 \frac{k}{2}$ haplotypes also (not even counting $h_{t}$ ).

As a result we have polynomial-time solvability of $M P P H(*, 2)-C 1$. 
Theorem 6. $M P P H(*, 2)$ is solvable in polynomial time if the compatibility graph is a clique.

\section{Postlude}

There remain a number of open problems. The complexity of $P H(*, 2)$ and $M P P H(*, 2)$ is still unknown. An approach that might raise the necessary insight is studying $P H(*, 2)-C k$ and $M P P H(*, 2)-C k$ variants of these problems (i.e. where the compatibility graph is the sum of $k$ cliques) for small $k$.

Another intriguing open question concerns the relative complexity of $P H$ and $M P P H$ instances. Has $P H(k, l)$ always the same complexity as $M P P H(k, l)$, in terms of well-known complexity measurements (polynomial-time solvability, NP-hardness, APX-hardness)? For hard instances, do approximability ratios differ? There do not yet exist any approximation algorithms for $M P P H$ and an immediate question is whether the weak $2^{k-1}$ approximation ratio for $P H$ can be attained (or improved) for $M P P H$. A related question is whether it is possible to directly encode $P H$ instances as $M P P H$ instances, and/or vice-versa, and if so whether/how this affects the bounds on the number of 2's in columns and rows.

For hard $P H(k, l)$ instances it would also be interesting to determine if the $2^{k-1}$ approximation ratio can be improved for fixed $l$. Finally, with respect to $M P P H$, it could be good to explore how parsimonious the solutions are that are produced by the various $P P H$ feasibility algorithms, and whether searching through the entire space of PPH solutions (as proposed in [18]) yields practical algorithms for solving $M P P H$.

\section{References}

1. Alimonti, P., Kann, V., Hardness of approximating problems on cubic graphs, Proceedings of the Third Italian Conference on Algorithms and Complexity, 288298 (1997)

2. Bafna, V., Gusfield, D., Hannenhalli, S., Yooseph, S., A Note on Efficient Computation of Haplotypes via Perfect Phylogeny, Journal of Computational Biology, 11(5), pp. 858-866 (2004)

3. Blair, J.R.S., Peyton, B., An introduction to chordal graphs and clique trees, in Graph theory and sparse matrix computation, pp. 1-29, Springer (1993)

4. Bonizzoni, P., Vedova, G.D., Dondi, R., Li, J., The haplotyping problem: an overview of computational models and solutions, Journal of Computer Science and Technology 18(6), pp. 675-688 (2003)

5. Brown, D., Harrower, I., Integer programming approaches to haplotype inference by pure parsimony, IEEE/ACM Transactions on Computational Biology and Informatics 3(2) (2006)

6. Cilibrasi, R., Iersel, L.J.J. van, Kelk, S.M., Tromp, J., On the Complexity of Several Haplotyping Problems, Proceedings of the 5th International Workshop on Algorithms in Bioinformatics (WABI 2005), LNBI 3692, Springer Verlag, Berlin, pp. 128-139 (2005) 
7. Ding, Z., Filkov, V., Gusfield, D., A linear-time algorithm for the perfect phylogeny haplotyping (PPH) problem, Journal of Computational Biology, 13(2) pp. 522-533 (2006)

8. Gusfield, D., Algorithms on Strings, Trees, and Sequences: Computer Science and Computational Biology, Cambridge University Press (1997)

9. Gusfield, D., Efficient algorithms for inferring evolutionary history, Networks 21, pp. 19-28 (1991)

10. Gusfield, D., Haplotype inference by pure parsimony, Proc. 14th Ann. Symp. Combinatorial Pattern Matching, pp. 144-155 (2003)

11. Halldórsson, B.V., Bafna, V., Edwards, N., Lippert, R., Yooseph, S., Istrail, S., A survey of computational methods for determining haplotypes, Proc. DIMACS/RECOMB Satellite Workshop: Computational Methods for SNPs and Haplotype Inference, pp. 26-47 (2004)

12. Lancia, G., Pinotti, M., Rizzi, R., Haplotyping populations by pure parsimony: complexity of exact and approximation algorithms, INFORMS Journal on Computing 16(4) pp. 348-359 (2004)

13. Lancia, G., Rizzi, R., A polynomial case of the parsimony haplotyping problem, Operations Research Letters 34(3) pp. 289-295 (2006)

14. Papadimitriou, C.H., Yannakakis, M., Optimization, approximation, and complexity classes, J. Comput. System Sci. 43, pp. 425-440 (1991)

15. Rose, D.J., Tarjan, R.E., Lueker, G.S., Algorithmic aspects of vertex elimination on graphs, SIAM J. Comput., 5, pp. 266-283 (1976)

16. Sharan, R., Halldórsson, B.V., Istrail, S., Islands of tractability for parsimony haplotyping, IEEE/ACM Transactions on Computational Biology and Bioinformatics, to appear

17. Song, Y.S., Wu, Y., Gusfield, D., Algorithms for imperfect phylogeny haplotyping (IPPH) with single haploplasy or recombination event, Proceedings of the 5th International Workshop on Algorithms in Bioinformatics (WABI 2005), LNBI 3692, Springer Verlag, Berlin, pp. 152-164 (2005)

18. VijayaSatya, R., Mukherjee, A., An optimal algorithm for perfect phylogeny haplotyping, Journal of Computational Biology, to appear

19. Xian-Sun Zhang, Rui-Sheng Wang, Ling-Yun Wu, Luonan Chen, Models and Algorithms for Haplotyping Problem, Current Bioinformatics 1, pp. 105-114 (2006) 